\title{
Providing a new approach to improve in speed and longevity of the Internet of Things based on RFID
}

\author{
Arman Kavoosi Ghafi \\ Department of Computer \\ Software, Central Islamshahr \\ Branch, Islamic Azad \\ University, Iran
}

\author{
Behnaz Farzi \\ Department of Computer, \\ Buinzahra Branch,Islamic Azad \\ University,Buinzahra,Iran
}

\author{
Helena Kojooyan Jafari \\ Department of Computer \\ Software, Central Islamshahr \\ Branch, Islamic Azad \\ University, Iran
}

\begin{abstract}
Identification Technology Using Radio Frequency tags (RFID) is a very advanced technology that is fairly named the greatest revolution after the Internet. Internet of Things is based on this technology and, it will be rapidly prevailed. A set of constraints that lie ahead is the major challenges of development and application of RFID networks. One of the most fundamental concerns is tag Readers optimal deployment in large-scale RFID network planning (RNP), which leads to optimal performance and increase in lifetime and speed of network. With considering coverage, signal interference and load balance as optimization targets and determination of optimum, the establishment issue of tag reader is converted to compound multi-objective optimization problem. In this article, in order to find the answers of the problem, the particle swarm algorithm (PSO) Combination with multi-objective optimization, based on Pareto's theory MOPSO that is able to solve the problem with more than one objective, was used. Simulation results show that the algorithm MOPSO compared to the optimization algorithms coverage, signal interference and load balancing has been effective. Therefore, with optimal deployment of tag readers, overall performance of system is improved.
\end{abstract}

Keywords: RFID, MOPSO, Internet of things, lifetime, tag readers.

\section{INTRODUCTION}

In large-scale RFID network systems, fast and efficient collection of information stored on the tags has a great importance, regarding the usage of applications, but this problem is not yet been fully investigated, and the comprehensive solution for it has not been presented yet. Due to limitations of domain and coverage range of tag readers, the tag readers should be in a compact format, in order to completely coverage a certain area, and to use minimum number of tag reader that is impossible. There are some problems, which are imposed on the deployment of RFID network that can be cited as an example: to determine the number of deployed readers, seating and setting parameters for each reader that the issue of planning network (RFID RNP) in RFID with large scale at an optimal level, has seriously challenged. The RNP problem is a large-scale nonlinear optimization problem with many variables, constraints and objectives proven that the RNP is a NP-Hard problem. Although the major factors in RNP is deploying reader for maximum coverage. Read speed of information and the RFID network longevity is one of the major concerns that must be considered. Because if the reader and tags are considered identical, assuming a uniform statistical distribution of tags in a network environment, Obviously, balancing the load between the readers, for example scope and balanced coverage volume, can lead to increased network speed and longevity. Accordingly in this article, with regard to coverage, interference signals and load balancing as a optimization goal, first of all we explain the inferential model of multi-objective optimization, and then using MOPSO improved algorithms, will be clarified, in order to have an increasing rate in the speed and life length of internet of things based on RFID network .

\section{Introduction of RFID}

RFID is the concept for automatic identification of an object using radio signals -based storage and retrieval of remote data (Clauberg et al., 2013). Generally, RFID technology is helping of the following equipment to implement:

1. Tag

2. Tag Reader

3. Antenna - Signal Booster

4. Information Management Software

5. Database

Radio waves carry information between sender and receiver information devices. To the sender piece of information, tag and to receiver pieces of information, called a reader or tag reader. Labels often lie on the object. If the tags are classified according to their energy sources that are being used, we have three main types of them. Active, passive and semi-passive Tags. Active and passive tags have many differences, But it can be noted that active tags, receives Their energy needs from their mobile battery, While passive tags itself has not been a source of energy and for tripping should use the energy of electromagnetic waves emitted by the tag reader, and their range and scope of read is less than active tags (Finer et al., 2011). Passive tags are low cost and long lifetimes and also small dimensions. Semi-passive tags is also another type of label that in addition to using its internal battery, can also use wave energy emitted by the tag reader. Antenna is used to transmit radio signals between the tag reader and tag that is used both for Tags and tag reader. There is a Data management software in order to process the collected data. This special software is usually on a local server that allows 
the exchanged Data with the tag reader, to be collected and processed, and stored in a database, and also be restored if needed. RFID technology can be an alternative for the barcodes. In fact, RFID is more than just a bar code, because it has an automatic system scanner. This two technologies have major differences. The two main differences can be elaborated in the following ways; RFID technology is capable of carrying large volumes of information, and also it does not need to have line of sight for data collection and communication. (Hvlmyvyst and Stephenson, 2006).

\section{Introduction of the Internet of Things}

The report prepared by the analysts of policy and strategies unit of ITU, has a look at the next step in a continuous and always ship (ITU, 2005). Based on this the new technologies, such as RFID, smart calculation promising, world of networked, and interconnected equipment. At that time, everything from car cyclic to brush come into communications area that is the announcer of rising in a new era, and will lead current Internet (which contains data and people) to the Internet of Things.

\section{Method}

\subsection{Optimization of Tag readers' deployment}

In this section, the first theoretical topics of issue, the formula array, and then the proposed algorithm, based on PSO will be presented.

\section{Problem formulation}

In large-scale RFID applications systems, three essential limitations should be considered comprehensively; increase overall performance and efficiency, prevention of data loss of labels, or tags which undoubtedly is unacceptable. In this field of study, Coverage is the main goal and the other two are directly related to this problem.

\section{A) The maximum coverage}

In the traditional method of modeling coverage rates, Cover Rate is obtained by dividing the number of covered tags toward the total number of tags. If we assume that $\mathrm{T}$ is a set of tags, based on coverage area, $\mathrm{R}$ be a set of tag readers, based by method or algorithm which is used in environment, $\mathrm{E}$ be the minimum threshold power of reliable receiving of ship between reader and tag, ER,T is most optimal power of tag, to get information of $\mathrm{T}$ member, tag by $\mathrm{r}$ tag reader that is a member of $\mathrm{R}$ collections. When we want to define the coverage area for $\mathrm{R}$ series tag readers, only the tags that the amount of their $E_{R, T}$ is higher than the minimum threshold of (E), and its similar amount is for any other of tag reader, in this case, it has better conditions than other tag readers which are taken into account in its range. Tags coverage area $\mathrm{R}$ can be applied as the number of tags that can be expressed in the following equation:

$C_{T}(R)=\left\{T \in T S \quad \mid E_{R, T} \geq E, E_{R, T} \geq E, E_{R^{\prime}, T}\left(\forall R^{\prime} \in R S, R^{\prime} \neq R\right)\right\}$

The optimization objective of coverage is the deployment of finite number of tag readers for full coverage area which is given below.

$$
T S=\bigcup_{R \in R S} c_{t}(R), \text { with minimize of }|R S|
$$

Therefore, the coverage rate can be defined as follows (equation (3)), that the goal is maximization of it:

$\operatorname{Max} f_{C R}=\frac{\sum_{R \in R S} C_{T}(R)}{|T S|}$

Where $\sum_{R \in R S} C_{T}(R)$ is the number of active tags used in the work area. $|T S|$ is the number of tags widespread in the environment. If $f_{C R}=1$, then the system has reached its optimal coverage.

\section{B) reduce signal interference}

Reduce signal interference can be considered as equivalent to a reduction of undesirable interaction of multiple tag readers. If interference or overlapping areas will increase by several readers as a result signal interference level will go up. So by reducing interference or overlapping areas, signal interference can also be reduced. Of course the number of tags based on overlapping regions has a significant impact on network performance. Assuming this to be done in this area of tag. But because of the presence or absence of tags, there is not prognosis in these areas. So for optimum director should be on reducing weaving or overlapping efforts. For each tag in a particular scene, if the power received by the other Tag readers be less than the best power $\mathrm{E}_{\mathrm{R}, \mathrm{T}}$, but larger than the minimum threshold of E, so $E_{R, T} \geq E_{R^{\prime}, T} \geq E$, then there is the possibility of signal interference for the tag. The level of signal interference for tags can be defined by $\varphi(T)$ and as follows:

$\varphi(T)=\sum\left(E_{R^{\prime}, T}-E\right)$, where $E_{R, T} \geq E_{R^{\prime}, T} \geq E$ Thus, for each tag, if the value of $E$ corresponding to the its overlying Tag readers be more than amount of E related to all other of Tag readers-based in environment, simply put, when $\max \left(\frac{E_{R, T}}{E_{R, T}+\varphi(T)}\right)$ is the maximum, can be sure that Signal interference with other tags does not happen in practice. With regard to the proposed, the goal function of reduce signal interference can be described as follows by equation (5):

$\max f_{s I}=\frac{\sum_{T \in T S}\left(\frac{E_{R, T}}{E_{R, T}+\varphi(T)}\right)}{|T S|}$ 
So when $f_{S I}=1$ and to reach its maximum value, the system reaches its optimum level in terms of reducing signal interference.

\section{C) Increasing the lifetime of the network with load balancing}

In line with the objectives mentioned above, set of observed tags should be covered properly and balanced by based tag readers. The ship between tag and its covering Tag readers via RF signal is carried out, used energy or power needed to any exchange of information between tag and tag reader with $C_{R, T}$ is shown. It is clear that the total communication cost or power consumption for each $\mathrm{R}$ tag reader will be as the sum of the total cost of communications coverage area, and defined as $\quad C_{R}=\sum_{R=\gamma(T)} C_{R, T} \quad$ which $R=\gamma(T)$ indicates that the $\mathrm{T}$ tag is covered by $\mathrm{R}$ tag reader. The aim of load balancing is finding an optimal distribution of tags to minimize the total power consumption $\left(C=\sum_{R \in R S} C_{R}\right)$ or is reduction of the cost and power consumption, which result in increasing of the network life time. To simplify our analysis, we put the amount of energy consumed for each exchange of information between tag and reader equal to one (Units). A simple system for energy consumption by equation (6) expressed as the number of tags by the reader $\mathrm{R}$ is covered

$$
\operatorname{Min} C=\sum_{R \in R S} C_{R}=\sum_{R \in R S} n_{R}
$$

The number of assigned tags are used to serve as a solution for load balancing, which is trying to minimize $\operatorname{Min} \Pi_{I=1}^{|R S|}\left(\frac{1}{n_{R(i)}}\right)$ and $n_{R(i)}$ is the number of tags covered by the (i) tag reader. If load balancing is the only goal of system optimization, this solution will have the best impacts and results. But, because there are a lot of variables that are fine. Its use does not seem useful for multi-objective programs. In this paper, according to information entropy theory, and the corresponding $S=-\sum P_{i} \log p_{i}$

Replace The $P$ value with $\frac{n_{R(i)}}{|T S|}$ between and define load balancing function with equation (7), where $|R S|$ represents the number of tags, and $|R S|$ represents the number of tag readers . To simplify calculations we rewrite equation (7) into equation (8), where $\operatorname{In}|R S|$ is used for ease of use and normalization. Once, it means that all tags are just balanced in covering readers. When $f_{L B}=1$, it means that all tags are just balanced in covering readers.
$\operatorname{Max} f_{L B}=-\sum_{i=1}^{|R S|} \frac{n_{R(i)}}{|T S|} \operatorname{In}\left(\frac{n_{R(i)}}{|T S|}\right)$

$\operatorname{Max} f_{L B}=-\sum_{i=1}^{|R S|} \frac{\frac{n_{R(i)}}{|T S|} \operatorname{In}\left(\frac{n_{R(i)}}{T S}\right)}{\operatorname{In}(|R S|)}$

\section{The proposed solution based on MOPSO}

As described in the previous section, in order to fulfill the formula functions of the three goals of the establishment of readers on the RFID systems at large scale, in order to identify and maximum coverage, and reduce interference and consequently increase network speed, as well as covering the balance to reduce adjusted cost and power consumption, and extend the life of the network, can be considered as the combination of optimized multi-objective optimization. PSO algorithm is a popular multi-dimensional optimization algorithm and is a proper technique for this purpose, the application of simple, high quality solutions and results, and efficient computing and high speed of convergence are PSO algorithm's strengths. PSO algorithm used at the position of each particle as a deployment solution is code reader, assuming $m=|R S|$ is the number of readers, the position of the particle $\mathrm{i}$ define as $x_{i}=\left\{x_{i, 1}, x_{i, 2}, \ldots, x_{i, 2 m}, x_{i, 2 m}\right\}$ in which each pair number represents the coordinates of a reader, so long $\left|x_{i}\right|=2 m$, mathematically speaking,

$$
\left(x_{i, 2 k-1,} x_{i, 2 k}\right) \in x_{i},(k=1 \ldots m)
$$

That reflects the position of coordinate's reader $\mathrm{k}$ in the i-th particle. Particle i fitness function (fitness (Xi)), is criterion for evaluating the position or solution of $\mathrm{Xi}$ in performance environments of network with $\mathrm{m}$ reader. In the proposed method the three objectives of functions that were formulated in the previous sections Instead of fitting function, we use than Pareto theory and we choose a set of optimal answer as the Pareto set.

$$
\left\{\begin{array}{l}
v_{i}^{(t+1)}=w v_{i}^{t}+c_{1} \gamma\left(p b_{i}^{(t)}-x_{i}^{(t)}\right)+c_{2} R\left(g b^{(t)-x_{i}^{(t)}}\right) \\
x_{i}^{(t+1)}=x_{i}^{(t)}+v_{i}^{(t+1)}
\end{array}\right\}
$$

In The PSO algorithm, each particle by a path with concept of timing of the allocation of resources be coded. The main idea of PSO is finding the best time of movement of the entire particle with the energy assessment, and implementation cost and time of implementation. Each particle contains a status indicating timing solutions and a velocity vector that represents the direction and amount of motion of the particle 
respectively. Position and speed of movement of the particles of the wishes expressed by the following expressions: Where $v_{i}^{t}$ represents the speed and $v_{i}^{t}$ represents the position of the particle $i$ at repetition $t$ and respectively $g b$ and $\mathrm{pb}$ are the best position or solution, and local (individual) and best overall (general). $\mathrm{r}$ and $\mathrm{R}$ are probabilistic random values in the range $[0,1]$ and $\mathrm{w}$ and $\mathrm{c} 1$ and $\mathrm{c} 2$ are weighting parameters.

To select the best local and global position, When more than one purpose in mind, the selection becomes a major challenge that To solve the problem we use of the concept of Pareto's theory to compare and choose the best timing or position of the particle in terms of the number of targets,. The process and the proposed algorithm is given below

\section{PSO multi-objective algorithm}

Step (1): we put value $\mathrm{t}=0$ for the first iteration. All $\mathrm{k}$ particles in the Stset community using the greedy algorithm are initialized.

Step (2): for each particle calculations using the equations (previous section) and the description of paragraph (2) of this section do and produce new particles $\mathrm{K}$ wishes. Total new and old particle forms $2 \mathrm{k}$ of community of Tset temporary memory.

Step (3): Using the theory of Pareto and formulated three objective function, we have found the optimized particles or the position of local optimization (Pbest $\mathrm{s}$ ) and it named with POset and we show the number of member of optimal set with Npo. If $\mathrm{Npo}<\mathrm{K}$ we go to step (4), otherwise the step (5) are going.

Step (4): we select the number of K-Npo particles from particles that are not optimal Pareto to add to the collection POset to complete a set number and give up to a number $\mathrm{K}$ to form set of particle ind The next step St+1set .

Step (5): we will select randomly $\mathrm{K}$ number of chosen Pareto optimal set of POset to create next step complex particle swarm St+1set set.

Step 6: Check the termination condition of the algorithm, consider that obtained conditions is favorable or the number of iterations has been completed or not. In case of termination condition go to step (8), otherwise the step (7) are going.

Step (7): The amount of $t=t+1$ up to date and to step (2) are going.

Step (8): algorithm Completed and we refer to Pareto optimal set of POset to decide and choose the nationwide optimal solution of Gbest.

\section{Results}

In this section will present the results of simulation using the proposed establishment of the reader MOPSO (multiobjective particle swarm algorithm), to analyze and compare the results thereof. These simulation was conducted by using MATLAB in 2011.

\section{Evaluation criteria}

Since the aim of this paper is to determine the location of readers on the RFID network to increase the speed and reduce the cost and increase longevity. And in this regard raised three main objectives that include increased maximum coverage in order to read the maximum number of tags and reliability of the network, Reduce signal interference in areas of overlap to reduce energy waste And thus extend the life of network and increase speed of network with correct and reliable connections Load balancing, or balance between reader and number and cost of tag readers Resulting in increased longevity and speed of the network because of the network secure connections. Thus for the performance evaluation of MOPSO proposed optimized algorithm , The secure relevance rate or lack of signal interference that is calculated by the number of tags identified by the total number of tags available in the network environment or the coverage, as the inverse probability function of the of relevance rate And to measure of load balancing of the logarithmic covered tags rat by any Tag reader to the total number tag readres In comparison to the number of tags, tag readers, reader coverage and algorithm parameters related to PSO (population, weighting coefficients, etc.) and multi-objective optimization (sink size and the number of objective functions, etc.) is used.

\section{Simulation parameters}

MATLAB programming languages is used to test and validate the proposed algorithm. PC specifications ci5 4 GB Intel processor and operating system Windows 7 was selected. The number of repeat elections was simulated 500 times, to simulate different scenarios were used to assess. The proposed algorithm with different values for the number of tags (200, 400,600 and 800) was tested. The size of the network environment was varies according to the number of tags and was calculated from equation $R^{2}=[0, \sqrt[2]{|T S|}] *[0, \sqrt[2]{|T S|}]$. For example, for the number of tags to 200, a circumference of $14 * 14$ was chosen to do so for the number of tags 400 tense environment, $20 \times 20$, for the number of tags 600 , dimensions network environment $24 * 24$ and to the end to the number of tags 800 twisted aspects of the environment $28 * 28$ were selected. Also experiment with different values of tag readers $(3,5,10, \ldots, 30)$ and evaluation of their role in the RFID system performance took place. All evaluations by three indicators related to the three objectives formulated, were tested. Other settings applied to simulate is given in the table (below).

Table 1. Simulation parameters 


\section{The simulation results}

\subsection{Overall assessment of the proposed algorithm}

In the proposed algorithm in the first step 200 tags into an area with the size of 14 in 14 randomly and evenly were distributed. Below figure shows the distribution of tags. Each tag has been shown in blue as small hollow circle. Tag readers with red + sign marked that the locations of them determined by algorithm-based recommendations MOPSO been. we repeat it for different values of tag and tag readers so that the results is given in the below picture.

\begin{tabular}{|c|c|}
\hline Value & The parameter \\
\hline $200,400,600,800$ & The number of tags \\
\hline $3-30$ & The number of tag readers \\
\hline $14,20,24,28$ & Dimensions of Network \\
\hline 2 & The minimum threshold power \\
\hline 10 & The maximum power of the tag readers \\
\hline 200 & Initial population of PSO algorithm \\
\hline 100 & Sink size for MOPSO \\
\hline 1 & C1 for PSO (personal learning \\
(coefficient
\end{tabular}

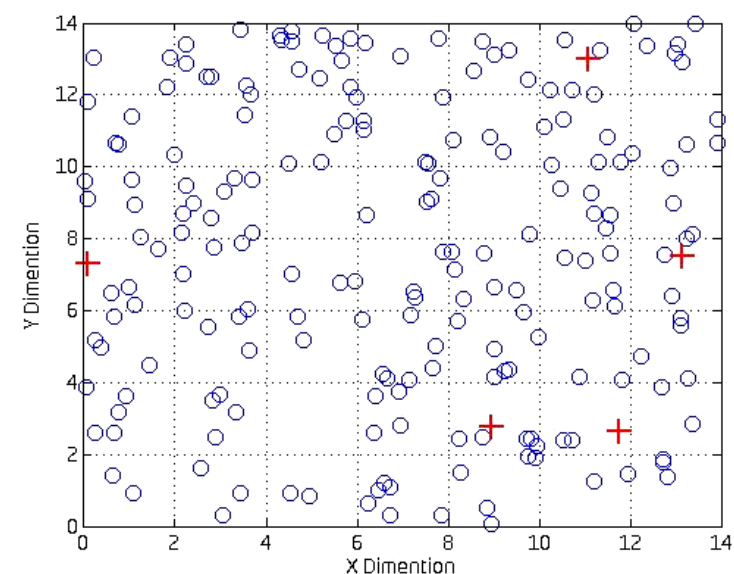

Figure 1: Distribution of 6 readers for random distribution network with 200 tags

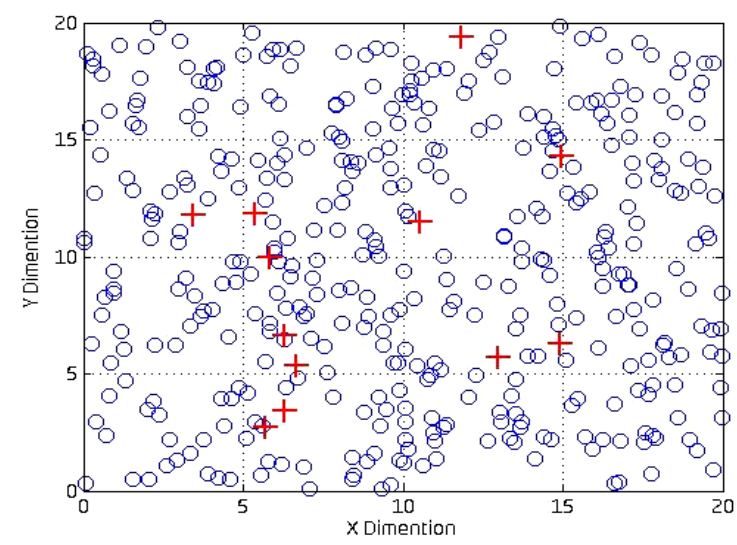

Figure 2: Distribution of 12 readers for random distribution of network with 400

tags

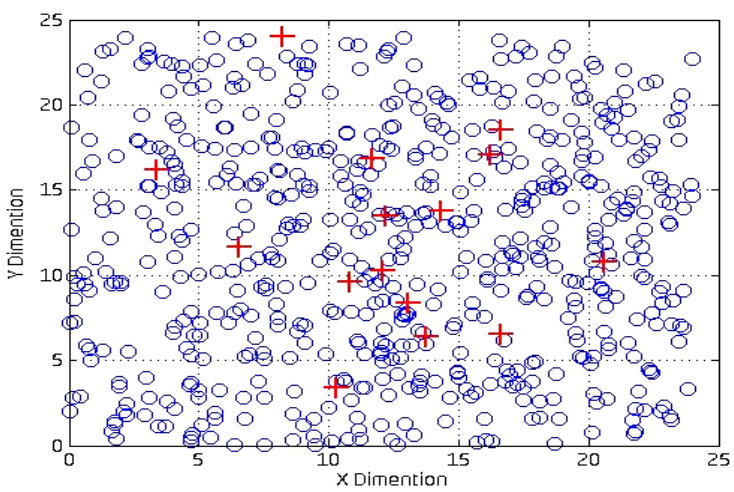

Figure 3: Distribution 15 tag reader for network with 600 tags by random distribution 


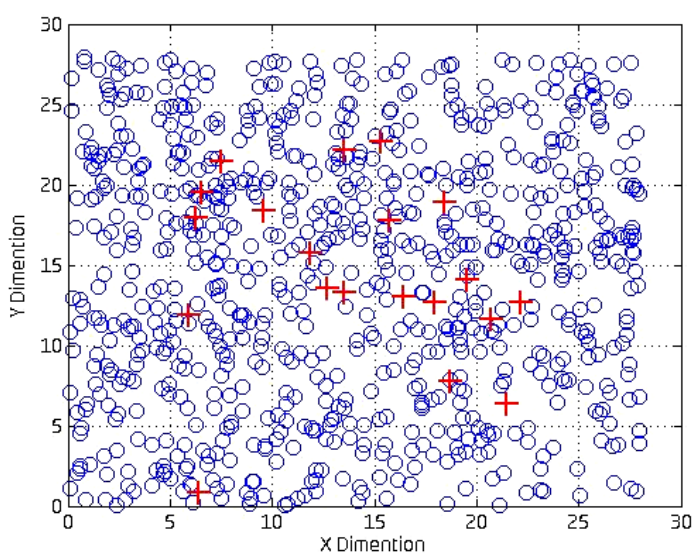

Figure 4: Distribution of 20 Tag reader for random distribution of network with 800 tags

\section{Efficiency assessment on the cover tags}

Since the basic objective of the proposed algorithm, is maximum coverage RFID tags by a reader network, at this stage of evaluation first the coverage of tags with different amounts of tag readers for a 200 number fixed number tag reader within the network environment Investigated discussed that the results is given in the table (below) and figure (below)

Table 2: The coverage rate of 200 tags by a different number for Tgkhvan

\begin{tabular}{|c|c|}
\hline Reader & Coverage \\
\hline 3 & 0.8 \\
\hline 5 & 0.865 \\
\hline 10 & 0.94 \\
\hline 12 & 0.965 \\
\hline 15 & 0.955 \\
\hline 17 & 0.97 \\
\hline 20 & 0.955 \\
\hline 23 & 0.96 \\
\hline 25 & 0.985 \\
\hline 27 & 0.96 \\
\hline 30 & 0.99 \\
\hline
\end{tabular}

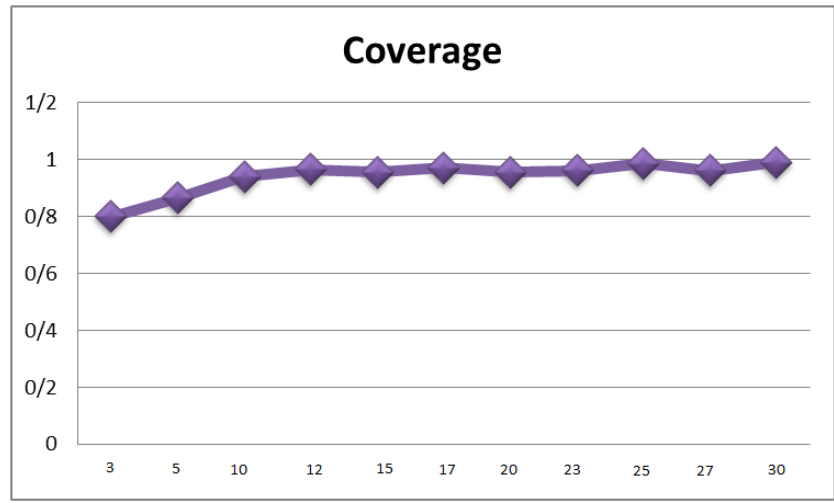

Figure 5: tags Coverage rate (tag number: 200) than the number of readers

In the figure (above), the horizontal axis and vertical axis represents the number of Tag reader and coverage rates in the range [01] shows. The table (above) and shape (above), it is observed that when the number of Tag reader that are less than 12 have coverage below $90 \%$. With the increasing of number of Tag readers, amount of coverage increased, but Tag reader increase from 10 to 30 does not make a significant difference in the rate of coverage, and it is inferred that to cover the $200 \mathrm{tag}$, in the RFID network environment with dimensions of $14 * 14$, the number of 10 to 12 Tag reader will suffice.

\section{Efficiency assessment to reduce signal interference and increase the speed and reliability of the grid}

The second purpose of this article was reduction of the maximum signal coverage, to evaluation the proposed method of image values derived from the formula used to signal interference. Therefore, the results obtained, show the rate of signal interference for different numbers Tag reader for a fixed number 200 indicates the number of tags in a network environment. The table (below) and form (below) shows the results.

Table 3: Rates of non-interference, tag of number of for Tag reader of 200

\begin{tabular}{|c|c|}
\hline Reader & $\begin{array}{c}\text { Signal } \\
\text { interference }\end{array}$ \\
\hline 3 & 0.99558 \\
\hline 5 & 0.99517 \\
\hline 10 & 0.99315 \\
\hline 12 & 0.99262 \\
\hline 15 & 0.99178 \\
\hline 17 & 0.99081 \\
\hline 20 & 0.99009 \\
\hline
\end{tabular}




\begin{tabular}{|c|c|}
\hline 23 & 0.98917 \\
\hline 25 & 0.98827 \\
\hline 27 & 0.98826 \\
\hline 30 & 0.98733 \\
\hline
\end{tabular}

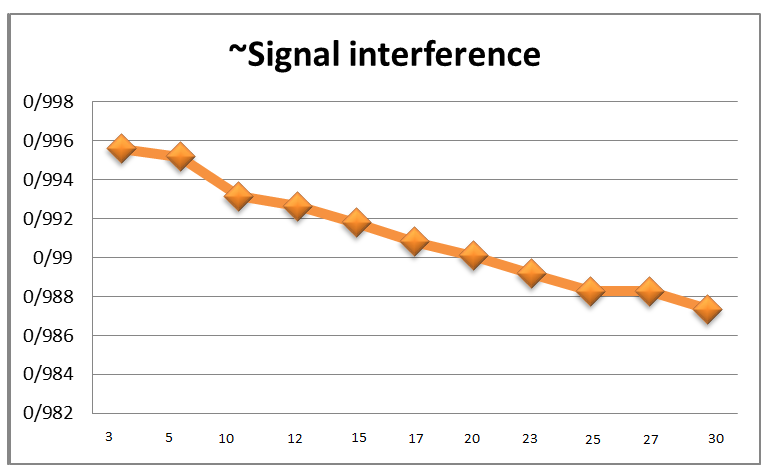

Figure 6: The amount of signal interference on the cover tags (tag of number of: 200) than the of number of readers

In the figure (above), the horizontal axis and vertical axis represents the number of Tag reader and load rates in [01] shows. Of the table (above) and shape (above), it is observed that when the number of Tag reader are less than 12 , the number of readers, rate of balance of the load increases significantly, but this amounts between 12 to 30 the difference is subtle number and the corresponding fluctuations could be due to random distribution is different in each simulation. The results also found that 11 to 12 pieces for readers on an area the size of $14 * 14$ and the most optimal tag of number of that are 200 .

\section{Efficiency assessment in the three goals compilation}

Figure (below) show The results of proposed algorithm to achieve three objectives defined in the multi-objective optimization Pareto collection, in the form (below) is observed that when of number of tags is 12 , the convergence caused in the three objectives (coverage, non-interference and load balance, and the optimum mode occurs, thus increasing more than this size is not necessary for the reader, and cause increasing cost. Therefore the best mode is intended for environments with tag of number of 200 number, is the 12 reader.

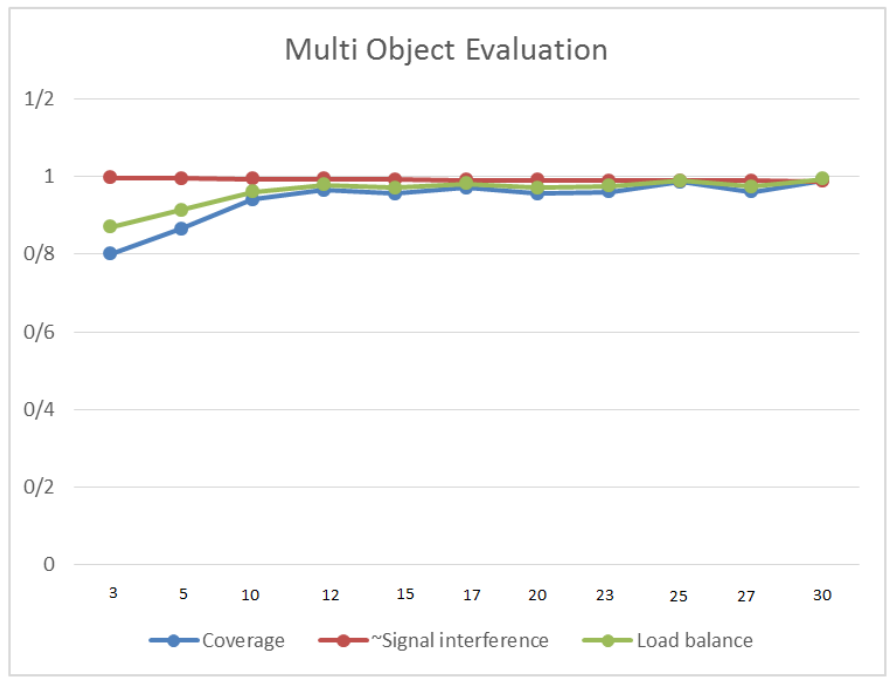

Figure 7: The three goals of efficiency rate (tag of number of: 200) than the of number of readers

\section{Evaluate and compare the proposed algorithm with previous approaches}

One of the important evaluations, is comparing the proposed algorithm with the previous method. In this evaluation of Ming Tao's, et al method in (2015) SA-PSO, Keyvan Kashkul Nejhad et al (2011) AFS and Mr. Lian Bo et al (2014) ABC for are chosen to be compared with the proposed method respectively. In the form (below), coverage rate are given for comparison algorithms. As can be seen in the figure, the proposed algorithm even in small numbers compared to the same Tag readers that are able to provide better coverage. When the number of readers is more than 10 the algorithms, the performance is similar, and difference is not tangible. And the proposed algorithm has been opened with a slight difference above the rest.

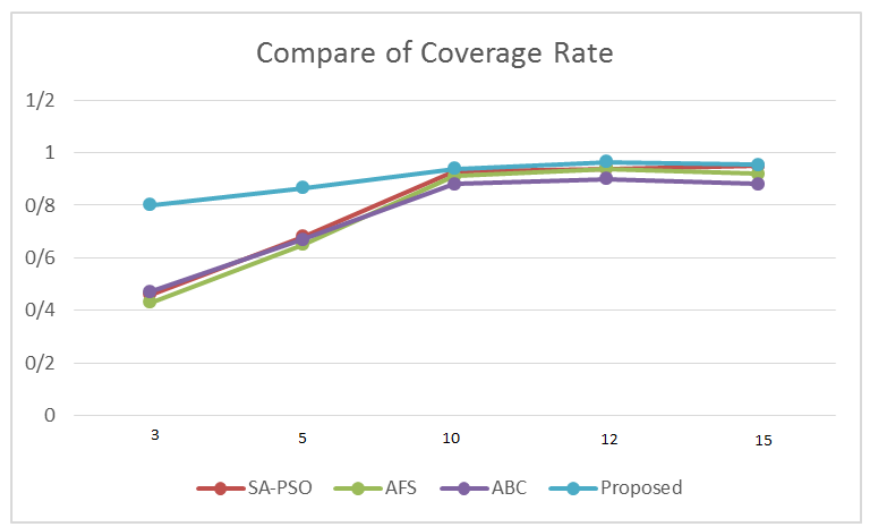

Figure 8: The algorithm coverage rates comparison to some readers

In the following comparison, the proposed algorithm in terms of solving the problem rate and reduce signal interference compared with the three previous algorithm. Figure (below) shows the results, in the figure $\mathrm{ABC}$ algorithms had the weakest performance, and AFS algorithm is in better shape. AFS performance and SA-PSO algorithm, and the proposed algorithm in the range of Tag reader number between 3 and 12 almost identical same performance and non-signal 
interference is of the top of $99 \%$, but when the number of readers increases the rate of non-interference in the AFS algorithm is reduced and by the end of this slope is proportional to the number Tag reader of decline. The SAPSO algorithm until that the number of tag readers are less than 23 acted feet the feet of the proposed algorithm in good condition and with the passage of 23 has been accelerated its decline and its slope is steeper. All algorithms by increasing tag readers their rate of non-interference is reduced, due to the increasing of levels of overlap and read a tag (which is located in the overlap region), At the same time is by two or more readers. But Tag reader improper placement can cause an increase in overlapping regions is resulting in increased interference. Increase the rate of non-interference evaluation index. According to the multi-objective algorithm and using Pareto multi-objective optimization theory could take several targets in conjunction And the use of indicators covering and interfering signals simultaneously, reduce signal interference and increase the rate of non-interference evaluation index.

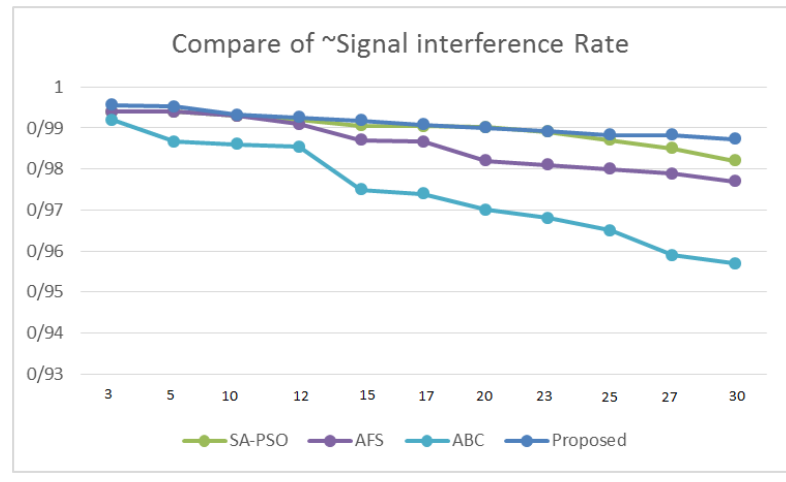

Figure 9: Comparison of the rate of signal interference algorithm to some readers

In the latest assessment, the rate of compliance with the proposed load balancing algorithm was tested in comparison with the three other algorithms. The third objective was to assess the use of the evaluation function. The figure (below) shows the results.

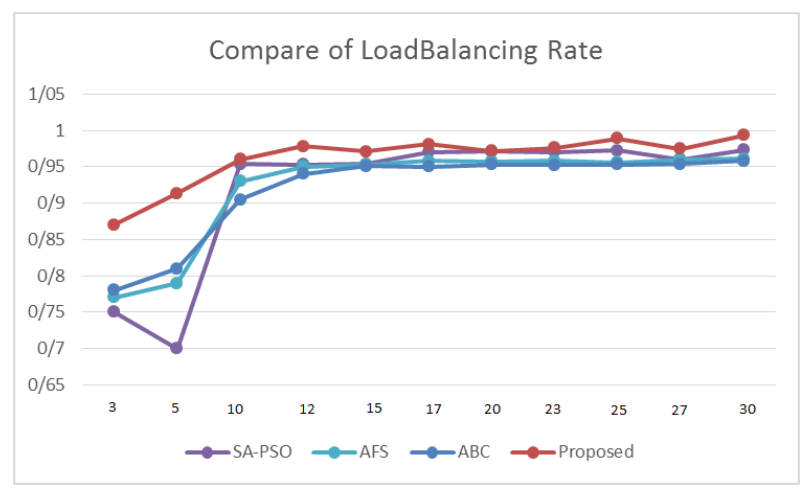

Figure 10: Comparison of the rate of load balancing algorithms to some readers

In figure (above), it is observed that when the number is less than 10 tag reader load balancing algorithms are weak in, in this range SA-PSO algorithm is the weakest mode. And the proposed algorithm is best achieved. ABC algorithm and AFS have same performance. But when the number of tag readers are more than 10 the Performance and smoothing algorithms are on a constant scope and are stable. Compliance rate balance of the proposed between algorithms is better than others, and between $97 \%$ and $99 \%$ each. Here, too, even at low doses cause of better proposed performance of settled algorithm is, using of multiple objective functions and power PSO algorithm at the same time.

\section{Conclusion}

In this section, the simulation results of the proposed algorithm to determine the optimal positioning and deployment of tag readers based on MOPSO algorithm in, the Internet of Things based on RFID network was presented. Then according to the diagram and simulation results are presented in tables, proved that the proposed algorithm has better performance in all aspects, especially when the number of readers is less than the proposed algorithm is better able to list and achieved good results. It was observed that excessive reader effecting improvement in three indicators and the extra cost is not raised. In addition excessive reader in a network environment with fixed dimensions increases more signals are overlapping areas. In total, according to the results, the efficiency of the proposed algorithm and method of applying meta-heuristic algorithm And the definition of beneficial triple objective functions and using Pareto multi-objective optimization theory concepts to determine the suitability solution or the particles proved Also revealed that the optimum MOPSO algorithm, has good feature set optimized for deployment reader prone position and has a network with more speed and long life.

\section{Research findings}

In this paper, a new algorithm for optimal deployment of tag readers for RFID networks, in order to extend the life and network speed are presented. The algorithm is actually a mapping which is based on three parameters: maximum coverage, reduce signal interference and increase load balancing. With the use of Particle Swarm (PSO) and multiobjective optimization Pareto's theory, that is formed by the combination is referred to as MOPSO, has tried to reduce overall cost, increase speed and increase the lifetime of the network, due to multi-objective fitness function. in PSO algorithm design frame talent place as the solution and particulate components were used as pair-pair And using the concepts of Pareto theory and multi-objective optimization from three angles and with creation a balance between them was achieved.

\section{Innovation research}

In this paper, try to pay in analysis of the RFID network information in Internet of Things. New aspects of the project is using analytical solutions in information networks of the Internet of Things in the event that That in the vast geographical expanse of massive amounts of data and data feeds used in data networks and also reduce the lifetime of the 
network. This paper aims to increase lifespan and maximum RFID tag detection in the world of the Internet of Things Taking advantage of a meta-heuristic methods to determine the position of the reader. The most important innovation in this article is using meta-heuristic algorithm in combination with multi-objective optimization PSO as defined MOPSO and efficient functions for these three purposes, which greatly increase the network coverage, reduce signal interference, increase the rate of load balancing and the thereby reducing the total cost of network speed and network lifetime and good connections.

\section{Suggestions}

As future work that can be done to develop a method presented in this paper, the following suggestions are presented:

- Use the proposed algorithm in wireless ad-hoc networks

- Use MOPSO algorithm considering deleted tag readers.

- Use Multi Objective Genetic Algorithm (MOGA) in the present case and compared with the method proposed in this paper.

- Applying another useful parameters for determining the fitness function.

- Use the proposed approach to integrated WSN and RFID network.

- Use other methods and hybrid approaches to determine the optimal number and position of their own tag locations (for example, using neural networks)

\section{REFERENCES}

[1] Clauberg, R., RFID and Sensor Networks, RFID Workshop, University of St. Gallen, Switzerland, Sept. 27, 2013.

[2] Fine, C., Klym, N., Trossen, D., and Tavshikar, M., The Evolution of RFID Networks: The Potential for Disruptive Innovation, MIT Center for eBusiness, CeB Research Brief, Vol. VIII, No. 1, 2011.

[3] Holmqvist, M., and Stefansson, G., Mobile RFID - A Case from Volvo on Innovation in SCM, System Sciences, HICSS '06. Proceedings of the 39th Annual Hawaii International Conference on, vol 6, IEEE, 2006.

[4] Ming Tao, ShuqiangHuang, YangLi, MinYan, YuyuZhou " SA-PSO based optimizing reader deployment in large-scale RFID Systems " journal homepage:

www.elsevier.com/locate/jnca.

[5] Keyvan Kashkouli Nejad, Xiaohong Jiang, Michitaka Kameyama. "High Performance Tag Singulation for Memoryless RFID Systems" This full text paper was peer reviewed at the direction of IEEE Communications Society subject matter experts for publication in the IEEE ICC 2011 proceedings.
[6] Lianbo Maa, b, Kunyuan Hua, Yunlong Zhua, Hanning Chena, "Cooperative artificial bee colony algorithm for multiobjective RFID network planning" Volume 42, June 2014, Pages 143-162. 